\title{
Aprendizagem Baseada em Problemas na formação médica e o currículo tradicional de Medicina: uma revisão bibliográfica
}

\author{
PALAVRAS-CHAVE \\ - Aprendizagem Baseada \\ em Problemas \\ - Educação médica/métodos \\ - Educação de graduação \\ em medicina
}

\section{KEY WORDS}

- Problem-Based Learning

- Education, medical/methods

- Education, medical, undergraduate

\section{Problem-Based Learning in medical education and the development of traditional medicine: a review of the literature}

Romeu Gomes ${ }^{\mathrm{I}}$

Rachel de Faria Brino ${ }^{I I}$

Aline Guerra Aquilante ${ }^{\mathrm{II}}$

Lucimar Retto da Silva de AvóII

\section{R E S U M O}

Este artigo analisa estudos que comparam a utilização da Aprendizagem Baseada em Problemas (ABP) na formação médica com o desenvolvimento de currículo tradicional de Medicina. O desenho metodológico, caracterizado como uma revisão da literatura sobre o assunto, realizada a partir de uma abordagem qualitativa, apoia-se na busca de artigos em bases de dados e bibliotecas virtuais, publicados de 1998 a 2008, com os termos medicina, graduação, aprendizagem baseada em problemas e ensino tradicional. O material foi estudado a partir da técnica de análise de conteúdo temática. A discussão se desenvolve a partir dos resultados acerca do uso da ABP na graduação médica em comparação aos resultados de cursos de currículo tradicional. Conclui-se que o uso da ABP na graduação médica pode ser uma alternativa na implementação das diretrizes brasileiras para a formação médica.

\section{A B S T R A C T}

This article examines studies that compare the use of problem-based learning (PBL) in medical training with the development of the traditional medical curriculum. The methodological design, a literature review of the subject with a qualitative approach, was based on an article search in databases and virtual libraries, with publications from 1998 to 2008, using the terms "medicine", "undergraduate education", "problem-based learning" and "traditional education". The material was studied using thematic content analysis. The discussion is based on results with use of PBL in undergraduate medical training, compared to the traditional medical curriculum. In conclusion, PBL in undergraduate medical education can be an alternative to traditional Brazilian curricular guidelines for medical training.

Recebido em: 12/09/2008 Reencaminhado em: 18/02/2009 Aprovado em: 24/03/2009

REVISTA BRASILEIRA DE EDUCAÇÃO MÉDICA

\footnotetext{
${ }^{I}$ Fundação Oswaldo Cruz, Rio de Janeiro, Rio de Janeiro, Brasil.

${ }^{I I}$ Universidade Federal de São Carlos, São Carlos, São Paulo, Brasil.
} 


\section{INTRODUÇÃO}

Em vários países, a formação médica vem sendo discutida com vistas a adotar um currículo adequado à realidade dos sistemas de saúde. No Brasil, as diretrizes curriculares para os cursos de graduação em Medicina vêm acompanhando o contexto mundial de transformação de referenciais da educação e das políticas de saúde. Essas mudanças buscam uma nova orientação que possa contribuir para a formação do profissional que a sociedade contemporânea exige ${ }^{1}$. As discussões sobre a formação médica, além de se voltarem para a revisão dos conteúdos curriculares, também vêm repensando as metodologias de ensino no sentido de torná-las mais adequadas ao perfil do profissional que se quer formar.

A concepção da Aprendizagem Baseada em Problemas $(\mathrm{ABP})$ vem se destacando como proposta metodológica que pode responder aos anseios de mudança curricular dos cursos de Medicina no País e no cenário mundial. O debate em torno da ABP é longo. Há mais de 30 anos tal proposta vem sendo implementada, avaliada, criticada e defendida. A utilização dessa concepção pedagógica nos cursos de Medicina no mundo inteiro tem como referência as ideias originais de Barrows e Tamblyn².

Os princípios norteadores do modelo de ensino ancorado na $\mathrm{ABP}$ permanecem atuais quando comparados com posicionamentos contemporâneos acerca da formação profissional. Perrenoud ${ }^{3}$, por exemplo, ao tratar a clínica como o momento da construção de saberes, de certa forma, retoma esse modelo pedagógico para analisar a formação médica, regida por uma lógica que tenta romper com o acúmulo mecânico de informações propedêuticas antes da inserção dos futuros profissionais médicos em atividades da prática profissional. Segundo esse autor, na ABP, a formação ocorre por meio da resolução de problemas estruturados pelos professores para que os alunos, pouco a pouco, construam o conhecimento necessário à resolução de problemas reais no futuro.

No modelo pedagógico norteado pela ABP, busca-se, principalmente, fornecer ao estudante condições de desenvolver habilidades técnicas, cognitivas e atitudinais aplicáveis tanto para o cuidado dos pacientes, quanto para a manutenção da postura de estudar para aprender pelo resto da vida profissional. Nesse modelo, em que o foco do processo educativo está centrado no estudante, estimula-se a capacidade de autoformação, fomentada pela busca ativa de informações. O estudante é estimulado a construir ativamente sua aprendizagem, articulando seus conhecimentos prévios com os de outros estudantes do grupo, para a resolução de problemas selecionados para o estudo, visando ao desenvolvimento do raciocínio crítico, de habilidades de comunicação e do entendimento da necessidade de aprender ao longo da vida ${ }^{2}$.
Essa proposta pedagógica vem sendo avaliada por estudos e pesquisas de vários países, num amplo debate comparativo com o currículo tradicional de Medicina. A análise desses estudos pode servir como ponto de partida para o debate sobre as mudanças metodológicas a adotar para tornar os cursos de Medicina mais adequados às diretrizes curriculares do País. Esse debate pode tanto beneficiar a formação do médico, quanto contribuir para que se repense a inserção desse profissional na área da saúde.

A partir dessa perspectiva, este trabalho tem por objetivo analisar estudos que comparam a utilização da Aprendizagem Baseada em Problemas na formação médica com o desenvolvimento de currículo tradicional na formação médica.

\section{METODOLOGIA}

Foi realizada uma busca de artigos nas bases de dados Lilacs, Medline e nas bibliotecas Cochrane e Scielo, sendo que o último acesso ocorreu em 2 de dezembro de 2008. Foram utilizados os termos medicina, graduação, aprendizagem baseada em problemas (ABP) e ensino tradicional, bem como seus correlatos em inglês. Com esses termos foram encontrados 91 artigos. Entre esses, foram selecionados os estudos que atendessem os seguintes critérios de inclusão: trabalho publicado no período de 1998 a 2008 e estudo que comparasse o ensino de graduação médica que utiliza a $\mathrm{ABP}$ com aqueles do currículo médico tradicional. Com base nesses critérios, foram identificados 11 artigos, dos quais quatro foram excluídos por tratarem apenas de uma área específica do curso de Medicina. Assim, ficaram sete artigos. Além das bases de dados e bibliotecas mencionadas, foi consultada a revisão sistemática de Koh et al. ${ }^{4}$, publicada em 2008. Selecionaram-se os estudos revisados, publicados de 1998 a 2008, considerados de alta qualidade pelos autores da revisão, sendo identificados seis, dos quais dois já tinham sido selecionados na Medline. Iniciada a análise, observou-se que dois artigos tratavam de um mesmo estudo: um focalizava os resultados de questionários respondidos por egressos de Medicina e outro por supervisores. Assim, este estudo analisa dez artigos.

Os artigos selecionados foram submetidos a uma primeira leitura, para que houvesse uma compreensão global dos estudos e para caracterizá-los quanto ao ano de publicação, método utilizado e país em que foram realizados.

Em seguida, foi feita uma análise qualitativa dos conteúdos das comparações realizadas pelos estudos entre a formação médica baseada na APB e aquela com currículo tradicional.

Em relação à análise qualitativa, trabalhou-se com uma adaptação da técnica de análise de conteúdo ${ }^{5}$, modalidade temática, descrita por Bardin ${ }^{6}$. Nessa análise, foram percorridos os seguintes passos: (a) identificação das ideias centrais presentes nas apresentações e discussões dos resultados dos estudos; (b) iden- 
tificação de núcleos de sentido subjacentes às ideias; (c) comparação entre os sentidos atribuídos aos resultados dos cursos de ABP e aqueles do currículo tradicional.

\section{Caracterização dos estudos analisados}

Em sua maioria, os estudos utilizaram metodologias quantitativas e foram publicados nos últimos quatro anos, sendo, portanto, investigações recentes (Quadro 1). Além disso, são provenientes de três países: Inglaterra, Estados Unidos e Canadá, sendo apenas um realizado na Austrália
Entre os dez estudos analisados, um americano ${ }^{10} \mathrm{e}$ um canadense ${ }^{14}$ avaliaram a transição de um currículo tradicional para um currículo com Aprendizagem Baseada em Problemas, sendo que o canadense diferia do americano por ser um currículo ABP orientado à comunidade.

Outro estudo, de coorte, cujos resultados foram publicados em dois artigos, comparou duas turmas do método tradicional com duas turmas do método ABP em relação a 31 competências e habilidades. Um dos artigos apresenta resultados referentes aos supervisores dos recém-formados durante a residência, após a conclusão da graduacão ${ }^{15}$. o outro focaliza os resultados em re-

Quadro 1

Características dos estudos analisados

\begin{tabular}{|c|c|c|c|c|}
\hline Estudo & País & Ano & Método Utilizado & Foco Central da Discussão \\
\hline $\begin{array}{l}\text { Crandall } \\
\text { et al. }{ }^{7}\end{array}$ & EUA & 2005 & Coorte & $\begin{array}{l}\text { Comparação do comportamento dos graduandos do currículo } \\
\text { tradicional e dos de ABP em relação às atitudes para com os } \\
\text { pacientes indigentes no decorrer do curso médico. }\end{array}$ \\
\hline $\begin{array}{l}\text { Distlehorst } \\
\text { et al. } 8\end{array}$ & EUA & 2005 & Estudo transversal & $\begin{array}{l}\text { Comparação do desempenho dos estudantes de uma única } \\
\text { instituição com dois currículos diferentes: tradicional e ABP. }\end{array}$ \\
\hline Hill et al. ${ }^{9}$ & Austrália & 1998 & $\begin{array}{l}\text { Ensaio } \\
\text { randomizado }\end{array}$ & $\begin{array}{l}\text { Comparação de como os estudantes de escolas médicas } \\
\text { tradicionais e não tradicionais se sentem em relação ao } \\
\text { preparo para a prática médica hospitalar. }\end{array}$ \\
\hline $\begin{array}{l}\text { Hoffman } \\
\text { et al. }{ }^{10}\end{array}$ & EUA & 2006 & Coorte & $\begin{array}{l}\text { Avaliação da transição de um currículo tradicional para um } \\
\text { currículo ABP. As avaliações foram realizadas tanto na } \\
\text { graduação quanto no primeiro ano de residência. }\end{array}$ \\
\hline $\begin{array}{l}\text { Jones } \\
\text { et al. }{ }^{11}\end{array}$ & Inglaterra & 2002 & Coorte & $\begin{array}{l}\text { Comparação da percepção dos recém-graduados e de seus } \\
\text { supervisores em relação a conhecimento, habilidades e atitudes } \\
\text { de estudantes advindos do currículo tradicional e ABP. }\end{array}$ \\
\hline $\begin{array}{l}\mathrm{O}^{\prime} \text { Neill } \\
\text { et al. }{ }^{12}\end{array}$ & Inglaterra & 2003 & Qualitativo & $\begin{array}{l}\text { Comparação da visão dos pré-residentes do curso tradicional } \\
\text { e ABP em relação ao seu grau de preparo para lidar com } \\
\text { incidentes críticos. }\end{array}$ \\
\hline $\begin{array}{l}\text { Peters } \\
\text { et al. }{ }^{13}\end{array}$ & EUA & 2000 & $\begin{array}{l}\text { Teste randomizado } \\
\text { controlado }\end{array}$ & $\begin{array}{l}\text { Comparação do método tradicional e ABP, segundo os } \\
\text { estudantes, em relação a comportamentos e atitudes } \\
\text { relacionados a três "domínios": medicina humanística, } \\
\text { aprendizagem ao longo da vida e aprendizagem social. }\end{array}$ \\
\hline $\begin{array}{l}\text { Tamblyn } \\
\text { et al. }{ }^{14}\end{array}$ & Canadá & 2005 & Coorte & $\begin{array}{l}\text { Avaliação da transição de um currículo tradicional para um } \\
\text { currículo ABP orientado à comunidade. O estudo foi realizado } \\
\text { só com médicos de saúde da família e não assalariados. }\end{array}$ \\
\hline $\begin{array}{l}\text { Watmoug } \\
\text { et al. }{ }^{15,16}\end{array}$ & Inglaterra & 2006 & Coorte & $\begin{array}{l}\text { Comparação de duas turmas do método tradicional e duas } \\
\text { turmas de ABP em relação a } 31 \text { habilidades, atitudes e } \\
\text { competências, segundo a ótica dos egressos de Medicina e } \\
\text { supervisores desses egressos no "estágio" após a conclusão da } \\
\text { graduação. }\end{array}$ \\
\hline $\begin{array}{l}\text { Willis } \\
\text { et al. }{ }^{17}\end{array}$ & Inglaterra & 2003 & Qualitativo & $\begin{array}{l}\text { Comparação das atitudes com a equipe de trabalho de } \\
\text { estudantes do curso médico tradicional e ABP. }\end{array}$ \\
\hline
\end{tabular}


lação aos próprios recém-formados ${ }^{16}$. Esse desenho de método foi utilizado por outro estudo inglês ${ }^{11}$, do tipo coorte, que também comparou a percepção de supervisores e recém-graduados em relação a conhecimento, habilidades e atitudes de estudantes advindos do currículo tradicional e ABP, utilizando as mesmas 31 competências e habilidades focalizadas no estudo anterior.

Em três estudos, os participantes eram os próprios estudantes. Um estudo australiano 9 de ensaio randomizado comparou, entre os estudantes de escolas médicas tradicionais e escolas com $\mathrm{ABP}$, como os estudantes se sentiam em relação à prática médica hospitalar. Um estudo inglês ${ }^{12}$, com metodologia qualitativa, também comparou, entre pré-residentes do curso tradicional e de $\mathrm{ABP}$, o quanto eles se sentiam preparados para lidar com incidentes críticos. E um estudo americano ${ }^{13}$, de teste randomizado controlado, fez a mesma comparação quanto a comportamentos e atitudes relacionados a três domínios: medicina humanística, aprendizagem ao longo da vida e aprendizagem social, sob a ótica dos próprios estudantes.

Há ainda a comparação de comportamentos dos estudantes de currículo tradicional e de $\mathrm{ABP}$ em relação a pacientes indigentes em um estudo americano de coorte ${ }^{7}$. Outro estudo americano ${ }^{8}$ compara, em uma mesma instituição, o desempenho dos estudantes advindos dos currículos tradicional e ABP. E, por fim, mais um estudo inglês, utilizando metodologia qualitativa, compara as atitudes dos estudantes dos dois currículos em relação à equipe de trabalho ${ }^{17}$.

\section{RESULTADOS}

Em geral, observa-se que os resultados dos cursos com ABP são mais positivos do que os dos cursos com o currículo tradicional (Quadro 2). Dos dez estudos, apenas os de Jones et al ${ }^{11} \mathrm{e}$ de Watmough et al ${ }^{15}$ e Watmough et al ${ }^{16}$ observaram que os cursos com ABP tiveram resultados piores do que os do currículo tradicional, mesmo assim em poucas competências e habilidades. Junto a isso, destaca-se que, em várias competências e habilidades, não houve diferença estatística significativa entre os resultados dos dois tipos de curso.

No que se refere aos resultados positivos dos cursos com $\mathrm{ABP}$, observa-se que os graduados se sentiram mais preparados ou foram avaliados mais positivamente por seus supervisores do que os graduandos dos cursos tradicionais, principalmente na dimensão social. Nessa dimensão, destacam-se de forma recorrente as competências de: comunicar-se de forma eficiente $^{10,11,16}$; efetivar relacionamento interpessoal ${ }^{9,10}$; lidar com pacientes de culturas diferentes ${ }^{11,16}$; levar em conta os aspectos psicossociais no adoecimento e no tratamento ${ }^{11,13,15}$; atuar em equi- $\mathrm{pe}^{9,10,11,15}$ e lidar com questões éticas ${ }^{11,15,16}$. Koh et $a l^{4}$, com base em sua revisão sistemática, constataram que a $\mathrm{ABP}$ desenvolvida nas escolas de Medicina tinham efeitos positivos na competência médica após a graduação, principalmente na dimensão social.

No conjunto dos resultados dos cursos de ABP mais bem avaliados que os cursos tradicionais, destacam-se também competências relacionadas à prevenção de doenças e promoção da saúde ${ }^{9,14,15}$ e à compreensão da articulação entre a atenção primária e o hospital11,15,16. Essas competências, de certa forma, contribuem para que a atuação médica não se reduza à intervenção, nem ao sistema hospitalar. Na revisão sistemática de Koh et al ${ }^{4}$, essas competências, classificadas por esses autores na dimensão social, foram avaliadas mais positivamente nos cursos de ABP do que nos de currículo tradicional.

Ainda em relação aos resultados mais positivos nos cursos de $\mathrm{ABP}$ do que nos de currículo tradicional, destacam-se capacidades pessoais evidenciadas em mais de um estudo: autoaprendizagem e busca por conhecimento ${ }^{9,10}$; iniciativa ${ }^{10}$; lidar com a crítica e com limites pessoais ${ }^{10,12}$; aceitar responsabilidade ${ }^{10,}$; lidar com a incerteza ${ }^{11,12,16}$ e lidar com a saúde pessoal e bem-es$\operatorname{tar}^{11,15}$. Entre essas capacidades, foram classificadas como de forte nível de evidência nos cursos de ABP por Koh e colaboradores $^{4}$ as capacidades de responsabilidade, lidar com a incerteza e lidar com a saúde pessoal e bem-estar.

Quanto às competências técnicas que envolvem habilidades médicas específicas, cinco foram apontadas com avaliações mais vantajosas nos cursos de $\mathrm{ABP}$ do que nos de currículo tradicional, em mais de um estudo: realização de história clínica, diagnóstico físico e prescrição $0^{10,11,15}$; compreensão de princípios de medicina baseada em evidência ${ }^{11,15,16}$; uso da informática como ferramenta da pratica médica ${ }^{11,15,16}$; realização de sutura ${ }^{11,15}$ e uso do eletrocardiograma ${ }^{11,15}$. Dessas competências, foram classificadas como de forte evidência na revisão sistemática de Koh et al $^{4}$ as seguintes: diagnóstico exato; compreensão de princípios de medicina baseada em evidência; uso do eletrocardiograma.

Em relação às capacidades que não mostraram diferenças significativas entre os cursos de $\mathrm{ABP}$ e os de currículo tradicional, as de maior concordância entre os estudos foram: lidar com as próprias limitações ${ }^{11,15,16}$ (avaliada como positiva em dois estudos ${ }^{10,12}$ ); usar laboratório e outros serviços de diagnóstico ${ }^{11,15,16}$; elaborar registros exatos ${ }^{11,15,16}$; realizar ressuscitação cardiopulmonar ${ }^{11,15,16}$ e usar oxigenoterapia com segurança11,15,16.

Ainda quanto a diferenças estatisticamente não significativas entre o curso com ABP e o de currículo tradicional, destaca-se o achado do estudo de Distlehorst $e t a l^{8}$, que não encontrou 


\begin{tabular}{|c|c|c|c|}
\hline \multicolumn{4}{|c|}{$\begin{array}{l}\text { Quadro } 2 \\
\text { Baseada em Problemas e currículo tradicional }\end{array}$} \\
\hline \multirow[t]{2}{*}{ Estudo } & \multicolumn{3}{|c|}{$\begin{array}{l}\text { Resultados da Aprendizagem Baseada em Problemas (ABP) } \\
\text { em comparação com o currículo tradicional }\end{array}$} \\
\hline & Positivos & Negativos & Iindiferentes \\
\hline $\begin{array}{l}\text { Crandall } \\
\text { et al. }\end{array}$ & & & $\begin{array}{l}\text { Não houve diferenças significativas } \\
\text { entre os dois grupos em relação a } \\
\text { atitudes com os pacientes indigentes. }\end{array}$ \\
\hline $\begin{array}{l}\text { Distlehorst } \\
\text { et al. }{ }^{8}\end{array}$ & $\begin{array}{l}\text { Desempenhos mais bem avaliados em } \\
\text { ginecologia/obstetrícia, psiquiatria e } \\
\text { clínica médica como um todo, bem como } \\
\text { em relação a conhecimento e raciocínio } \\
\text { clínicos e comportamento não cognitivo. }\end{array}$ & & $\begin{array}{l}\text { Não houve diferença na } 1^{\underline{a}} \text { e } 2^{\underline{a}} \text { fases } \\
\text { do Exame de Licença Médica dos } \\
\text { Estados Unidos. }\end{array}$ \\
\hline Hill et al. ${ }^{9}$ & $\begin{array}{l}\text { Maior preparo em seis das oito áreas } \\
\text { avaliadas. }\end{array}$ & & $\begin{array}{l}\text { Não houve diferença em duas das } \\
\text { oito áreas avaliadas. }\end{array}$ \\
\hline $\begin{array}{l}\text { Hoffman } \\
\text { et al. }{ }^{10}\end{array}$ & $\begin{array}{l}\text { Melhores resultados dos graduandos na } \\
1^{\text {a }} \text { e } 2^{\underline{a}} \text { fases do Exame de Licença Médica. } \\
\text { Avaliações dos supervisores mais } \\
\text { positivas em } 12 \text { das } 17 \text { competências, no } \\
1^{\text {o }} \text { ano de residência médica. }\end{array}$ & & $\begin{array}{l}\text { Não houve diferença estatística } \\
\text { significativa em } 5 \text { das } 17 \\
\text { competências. }\end{array}$ \\
\hline $\begin{array}{l}\text { Jones } \\
\text { et al. }{ }^{11}\end{array}$ & $\begin{array}{l}\text { Graduados mais preparados em } 12 \text { das } 19 \\
\text { competências avaliadas e em } 8 \text { das } 13 \\
\text { habilidades específicas avaliadas. } \\
\text { Os supervisores consideram que os } \\
\text { graduados eram melhores do que os do } \\
\text { grupo controle em } 5 \text { das } 18 \text { competências } \\
\text { listadas. }\end{array}$ & $\begin{array}{l}\text { Graduados avaliaram } \\
\text { que o estudo do processo } \\
\text { da doença } \\
\text { (fisiopatologia) é melhor } \\
\text { no método tradicional. }\end{array}$ & $\begin{array}{l}\text { Não houve diferença entre as } \\
\text { respostas dos graduados e as do } \\
\text { grupo controle em relação a } 7 \text { das } 19 \\
\text { competências e em } 5 \text { das } 13 \\
\text { habilidades específicas avaliadas. } \\
\text { Segundo os supervisores, não houve } \\
\text { diferença nas } 13 \text { habilidades } \\
\text { específicas avaliadas. }\end{array}$ \\
\hline $\begin{array}{l}\mathrm{O}^{\prime} \mathrm{Neill} \\
\text { et } a l^{12}\end{array}$ & $\begin{array}{l}\text { Graduados sentiram-se mais bem } \\
\text { preparados para lidar com incertezas, } \\
\text { com seus limites e para tomar decisões na } \\
\text { pré-residência. }\end{array}$ & $\begin{array}{l}\text { Dificuldade de } \\
\text { comunicação e } \\
\text { envolvimento emocional. }\end{array}$ & \\
\hline $\begin{array}{l}\text { Peter } \\
\text { et al. }{ }^{13}\end{array}$ & $\begin{array}{l}\text { Avaliações dos graduados mais positivas } \\
\text { em } 3 \text { dos } 8 \text { aspectos do domínio da } \\
\text { medicina humanística, bem como em } 2 \\
\text { dos } 9 \text { aspectos do domínio da } \\
\text { aprendizagem social. }\end{array}$ & & $\begin{array}{l}\text { Não houve diferenças em } 4 \text { dos } 8 \\
\text { aspectos do domínio da medicina } \\
\text { humanística; nos } 4 \text { aspectos no } \\
\text { domínio da aprendizagem ao longo } \\
\text { da vida; e em } 7 \text { dos } 8 \text { aspectos do } \\
\text { domínio da aprendizagem social. }\end{array}$ \\
\hline
\end{tabular}




\begin{tabular}{|c|c|c|c|}
\hline \multicolumn{4}{|c|}{$\begin{array}{l}\text { Quadro } 2 \\
\text { Baseada em Problemas e currículo tradicional }\end{array}$} \\
\hline \multirow[t]{2}{*}{ Estudo } & \multicolumn{3}{|c|}{$\begin{array}{l}\text { Resultados da Aprendizagem Baseada em Problemas (ABP) } \\
\text { em comparação com o currículo tradicional }\end{array}$} \\
\hline & Positivos & Negativos & Iindiferentes \\
\hline $\begin{array}{l}\text { Tamblyn } \\
\text { et al. }{ }^{14}\end{array}$ & $\begin{array}{l}\text { Após a transição do currículo tradicional } \\
\text { para } \mathrm{ABP} \text {, houve aumento significativo } \\
\text { no cuidado preventivo e na continuidade } \\
\text { do cuidado, bem como melhora nos } \\
\text { indicadores de desempenho relativos ao } \\
\text { diagnóstico e à prescrição de } \\
\text { medicamentos. }\end{array}$ & & $\begin{array}{l}\text { Não foram encontradas diferenças } \\
\text { nas taxas de prescrição de } \\
\text { medicamentos contraindicados para } \\
\text { idosos. }\end{array}$ \\
\hline $\begin{array}{l}\text { Watmough } \\
\text { et al. }{ }^{15,16}\end{array}$ & $\begin{array}{l}\text { Avaliações dos graduados mais positivas } \\
\text { em } 16 \text { das } 31 \text { competências listadas. } \\
\text { Avaliações dos supervisores mais } \\
\text { positivas em } 15 \text { das } 31 \text { competências } \\
\text { listadas. }\end{array}$ & $\begin{array}{l}\text { Avaliações dos } \\
\text { graduados mais } \\
\text { negativas em } 2 \text { das } 31 \\
\text { competências. } \\
\text { Avaliações dos } \\
\text { supervisores mais } \\
\text { negativas em } 1 \text { das } 31 \\
\text { competências. }\end{array}$ & $\begin{array}{l}\text { Não houve diferenças nas avaliações } \\
\text { dos graduados em } 15 \text { das } 31 \\
\text { competências, listadas. } \\
\text { Não houve diferenças nas avaliações } \\
\text { dos supervisores em } 15 \text { das } \\
\text { competências listadas. }\end{array}$ \\
\hline
\end{tabular}

diferenças na primeira e segunda fase do Exame de Licença Médica dos Estados Unidos. Isto significa que o novo formato de formação médica, embora não tenha resultados superiores aos do formato tradicional, não pesou negativamente na certificação profissional de seus egressos.

Por último, observa-se que em três estudos os cursos com $\mathrm{ABP}$ tiveram resultados mais negativos do que os cursos tradicionais, nos seguintes quesitos de avaliação: conhecimentos em fisiopatologia ${ }^{11}$; comunicação ${ }^{12}$; envolvimento emocional ${ }^{12}$; entendimento do processo da doença ${ }^{15,16}$ e diagnóstico, tomada de decisão e tratamento (incluindo prescrição) ${ }^{15}$.

\section{DISCUSSÃO}

Assim como na revisão sistemática de Koh et al ${ }^{4}$, observou-se que a utilização da Aprendizagem Baseada em Problemas nas escolas de Medicina tem efeitos positivos após a graduação, principalmente no que se refere às competências da dimensão social. Entretanto, para uma validação mais apurada dos resultados dos estudos analisados, seria importante ter acesso a uma reflexão acerca dos fatores de processo que poderiam ter influenciado os resultados, uma vez que o entendimento e a implementação da ABP possivelmente variaram de curso para curso. Nesse sentido, seria importante ter não só uma avaliação de resultados, mas também uma análise dos diferentes processos. Para isso, seria importante que, no conjunto dos estudos analisados, houvesse mais estudos qualitativos, uma vez que esses estudos, como observam Crandall et $\mathrm{al}^{7}$, poderiam focalizar o que aconteceu ao longo dos cursos para compreender e comparar as características de estudantes cujas atitudes melhoraram ou declinaram durante a formação. Além disso, para se ter uma avaliação mais aprofundada dos efeitos desse modelo de formação, seria importante comparar o cotidiano da prática dos graduandos com o cotidiano da prática profissional dos egressos dos cursos tradicionais.

Os estudos trouxeram evidências de que certas competências recomendadas pelas diretrizes brasileiras sobre formação médica $^{1}$, a exemplo das relacionadas à área social, foram mais bem alcançadas nos cursos que adotaram o modelo pedagógico da $\mathrm{ABP}$ do que naqueles que seguem um modelo tradicional. A $\mathrm{ABP}$ pode ser um caminho para a participação ativa do aluno na construção do conhecimento e a integração entre os conteúdos, também recomendada pelas diretrizes curriculares.

Essa recomendação, entretanto, deve ser situada como uma alternativa e não como norma a ser seguida pelos cursos brasileiros de Medicina, uma vez que, como evidenciado nos diferentes 
estudos revisados, ainda são necessárias avaliações tanto dos resultados quanto do processo da adoção da ABP na formação médica. Por outro lado, ainda não há publicações de pesquisas de avaliação brasileiras que comparem curso ABP com curso de currículo tradicional. Isto indica a necessidade de realizar estudos para que os resultados internacionais desse novo formato de curso sejam validados em âmbito nacional.

No Brasil, Gomes et al ${ }^{18}$ avaliaram os resultados de uma experiência de curso ancorada na ABP. Com base em pesquisa com graduados em Medicina, pacientes e gestores dos graduados, apesar de pontuarem limites, esses autores apresentam resultados positivos dessa experiência. Relatam que, na ótica dos graduados, o curso proporcionou uma formação humanista, o aprender a aprender, a convivência com outros profissionais e a integração teoria-prática. Em relação aos pacientes e aos gestores entrevistados, destacam que os primeiros consideraram que os egressos eram cuidadosos, respeitando-os e escutando-os, enquanto os segundos reconheceram que a atuação do egresso ia além do foco da doença.

Os resultados exitosos e os limites desse estudo brasileiro podem servir de ponto de partida para a elaboração de hipóteses a serem investigadas em outros estudos nacionais, sobretudo de desenho caso-controle, para aprofundar mais a discussão acerca do uso da ABP na formação médica.

\section{CONCLUSÕES}

Com base na presente revisão, observa-se que o uso da ABP na graduação médica pode ser uma alternativa para a implementação das diretrizes brasileiras sobre a formação médica ${ }^{1}$. Entretanto, como observam Gomes et al ${ }^{18}$, a eficácia de cursos ancorados na ABP não depende apenas de ações eficientes relacionadas ao planejamento e à gestão curriculares. Depende também da articulação entre currículo e realidade profissional, para que haja reorientação de saberes e de práticas tanto no espaço acadêmico como fora dele, aí incluído o âmbito do sistema de saúde, em busca das mudanças que se pretende obter.

\section{REFERÊNCIAS}

1. Brasil. Ministério da Educação. Conselho Nacional de Educação. Câmara de Educação Superior. Resolução CNE/CES nº 4, de 7 de novembro de 2001. Institui diretrizes curriculares nacionais dos cursos de graduação em medicina. [documento na internet]. Brasília; 2001. [acesso em: 09 dez.2007]. Disponível em: http://portal.mec.gov.br/cne/arquivos/pdf/CES04.pdf.

2. Barrows HS, Tamblyn RM. Problem-Based Learning: an approach to medical education. New York: Springer; 1980.
3. Perrenoud P. A prática reflexiva no ofício de professor: profissionalização e razão pedagógica. Porto Alegre: Atmed; 2002.

4. Koh GCH, Khoo HE, Wong ML, Koh D. The effects of problem-based learning during medical school on physician competency: a systematic review. Can Med Assoc J. 2008; 178(1):34-41.

5. Gomes R. Análise e interpretação de dados de pesquisa qualitativa. In: Minayo MCS, org. Pesquisa social: teoria, método e criatividade. Petrópolis: Vozes; 2007. p.79-108.

6. Bardin L. Análise de conteúdo. Lisboa: Edições 70; 1979.

7. Crandall SJS, Reboussin BA, Michielutte R, Anthony JE, Naughton MJ. Medical students' attitudes toward underserved patients: a longitudinal comparison of Problem-Based and traditional medical curricula. Adv Health Sci Educ Theory Pract. 2007;12(1):71-86. DOI 10.1007/s10459-005-2297-1

8. Distlehorst LH, Dawson E, Robbs RS, Barrows HS. Problem-Based Learning outcomes: the glass half-full volume. Acad Med. 2005;80(3):294-9.

9. Hill J, Rolfe IE, Pearson SA, Heathcote A. Do junior doctors feel they are prepared for hospital practice? a study of graduates from traditional and non-traditional medical schools. Med Educ. 1998;32(1):9-24.

10. Hoffman K, Hosokawa M, Blake Jr. R, Headrick L, Johnson G. Problem-based learning outcomes: ten years of experience at the University of Missouri-Columbia School of Medicine. Acad Med. 2006;81(1):617-25. Doi: 10.1097/01.ACM.0000232411.97399.c6

11. Jones A, McArdles PJ, O'Neill PAO. Perceptions of how well graduates are prepared for the role of pre-registration house officer: a comparison of outcomes from a traditional and an integrated PBL curriculum. Med Educ. 2002; 36(1):16-25.

12. O'Neill PA, Jones A, Willis SC, McArdle PJ. Does a new undergraduate curriculum based on Tomorrow's Doctors prepare house officers better for their first post? a qualitative study of the views of pre-registration house officers using critical incidents. Med Educ. 2003;37(12):1100-1108

13. Peters AS, Greenberger-Rosovsky R, Crowder C, Block $\mathrm{SD}$, Moore GT. Long-term outcomes of the New Pathway Program at Harvard Medical School: a randomized controlled trail. Acad Med. 2000;75(5):470-9.

14. Tamblyn R, Abrahamowicz M, Dauphinee D, Girard N, Bartlett G, Grand Maison P et al. Effect of a community oriented problem based learning curriculum on quality of primary care delivered by graduates: historical cohort comparison study. $\mathrm{Br}$ Med J. 2005;331:1002-8. Doi:10.1136/bmj.38636.582546.7C 
15. Watmough S, Taylor DC, Garden A. Educational supervisors' views on the competencies of pre-registration house officers. Br J Hosp Med. 2006;67(2):92-5.

16. Watmough S, Ryland I, Taylor DC, Garden A. Pre-registration house officer skill and competency assessment through questionnaires. Br J Hosp Med. 2006; 67(9):487-90.

17. Willis S, Jones A, McArdle P, O’Neill PA. A qualitative study of the attitudes to teamwork of graduates from a traditional and an integrated undergraduate medical course. Adv Health Sci Educ Theory Pract. 2003; 8:139-148.

18. Gomes R, Bagnariolli AMF, Hamamoto CG, Moreira HMM, Costa MCG, Hafner MLMB et al. A formação médica ancorada na aprendizagem baseada em problema: uma avaliação qualitativa. Interface Comun Saúde Educ. [periódico na internet]. 2009;13(28):71-83. [Acesso em: 13 fev. 2009]. Disponível em: http:/ / www.interface.org.br/. Doi: 10.1590/S1414-32832009000100007

\section{CONTRIBUIÇÃO DOS AUTORES}

R. Gomes trabalhou na concepção do artigo, na pesquisa bibliográfica e na redação final do texto; A. G. Aquilante, R. de F. Brino e L. R. da S. de Avó trabalharam na pesquisa bibliográfica e na redação final do texto

\section{CONFLITO DE INTERESSES}

Declarou não haver

\section{ENDEREÇO PARA CORRESPONDÊNCIA}

Av. Rui Barbosa, 716

Flamengo - Rio de Janeiro

CEP.: 22250-020 RJ

E-mail: romeu@iff.fiocruz.br 